\title{
Gauge fields and interferometry in folded graphene
}

\author{
Diego Rainis, ${ }^{1, *}$ Fabio Taddei, ${ }^{1}$ Marco Polini, ${ }^{1,2}$ Gladys León, ${ }^{1,2}$ Francisco Guinea,${ }^{3,2}$ and Vladimir I. Fal'ko ${ }^{4,2}$ \\ ${ }^{1}$ NEST, Istituto Nanoscienze-CNR and Scuola Normale Superiore, I-56126 Pisa, Italy \\ ${ }^{2}$ Kavli Institute for Theoretical Physics China, CAS, Beijing 100190, China \\ ${ }^{3}$ Instituto de Ciencia de Materiales de Madrid (CSIC), Sor Juana Inés de la Cruz 3, E-28049 Madrid, Spain \\ ${ }^{4}$ Physics Department, Lancaster University, Lancaster LA1 4YB, United Kingdom
}

(Received 27 January 2011; published 5 April 2011)

\begin{abstract}
Folded graphene flakes are a natural byproduct of the micromechanical exfoliation process. In this article we show by a combination of analytical and numerical methods that such systems behave as intriguing interferometers due to the interplay between an externally applied magnetic field and the gauge field induced by the deformations in the region of the fold.
\end{abstract}

DOI: 10.1103/PhysRevB.83.165403

PACS number(s): 72.80.Vp, 72.10.Fk, 73.22.Pr, 73.23.-b

\section{INTRODUCTION}

Gauge fields modulate the dynamics and interactions of electrons in a variety of scales from particle cosmology to phase transitions in condensed matter. ${ }^{1}$ Graphene is a one-atom-thick carbon crystal where charge carriers behave as massless quasiparticles ${ }^{2}$ and where the impact of lattice deformations on electrons is equivalent to effective gauge fields. ${ }^{2-5}$ Graphene behaves as a highly stretchable membrane $^{6}$ whose elastic deformations can be induced in a controlled way. $^{7}$

A typical system in which such deformations occur is represented by a graphene fold [see Fig. 1(a)], that is, a single graphene flake that, as a result of mechanical or chemical procedures, is bent onto itself thereby creating two atomically close graphene layers lying on top of each other. Such folds are intentionally or accidentally created, for example, during the micromechanical exfoliation process that leads to the production of graphene monolayers. ${ }^{8}$ Depending on the rotational stacking fault which is introduced between the two layers, these can behave as decoupled monolayers. ${ }^{8,9}$ Despite the rather small interlayer distance (of the order of a few Angstroms), separate control of carrier densities and mobilities has been demonstrated. ${ }^{9}$ (See also Ref. 10 for a somewhat similar situation in which two ordinary two-dimensional electron gases give rise to coupled quantum Hall edge states in corner-overgrown heterostructures.)

In this article we study transport in a graphene fold subjected to the presence of an externally applied magnetic field. We fully take into account the impact of deformations in the region of the fold, which give rise to local gauge fields. We demonstrate that the interplay between the external magnetic field and the fold-induced gauge fields leads to the generation of peculiar chiral electronic modes which propagate parallel to the fold. As a result, pronounced quantum interference effects can be observed in this system because of the magnetic flux enclosed between the different paths of these chiral modes. The device allows for the controlled splitting of electronic current paths, opening the way for new graphene-based multiterminal (Mach-Zehnder) interferometers. ${ }^{11}$

Transport in graphene folds has already been studied by Prada et al. ${ }^{12}$ These authors have shown that in the quantum Hall regime electrons belonging to the "zero Landau level" (see below) are always perfectly transmitted through the fold: their study, however, completely neglects the role of the gauge fields present in the region of the fold, which are responsible for the main physical features we describe below.

The article is organized as follows. In Sec. II, we introduce the model, derive two contributions to the gauge field generated by the deformations at the fold, and show that peculiar chiral edge states are induced close to the fold. In Sec. III we analyze in great detail the edge-state configuration in the proximity of the fold and we show how such states can enclose a finite magnetic flux leading to interference effects. In Sec. IV we present our main numerical results on the transport properties of the system and show that interference indeed occurs giving rise to pronounced oscillations in the two-terminal conductance as a function of the external magnetic field. Finally, our main conclusions are summarized in Sec. V.

\section{MODEL SYSTEM}

We consider the setup sketched in Fig. 1(a). It consists of a graphene nanoribbon with armchair edges (AGNR), which has been folded along a line perpendicular to its longitudinal axis, here taken to be along the $\hat{\boldsymbol{x}}$ direction. We assume that the two layers are completely decoupled ${ }^{8,13,14}$ and that they are connected only through the region of the fold. Under these assumptions, current injected through a lead attached to the bottom layer, say, can be extracted from a lead attached to the top layer after having crossed the region of the fold.

The radius of the fold $R$ is determined by the balance between the bending rigidity of graphene $\kappa$ and the van der Waals attraction $\gamma$ between the two layers. ${ }^{15,16}$ We find (see Appendix A) $R \approx \sqrt{\kappa / \gamma} \approx 7 \AA$, where $\kappa \approx 1 \mathrm{eV}$ and $\gamma \approx 0.022 \mathrm{eV} \times \AA^{-2}$. The deformation associated with the fold also induces a uniaxial strain along the $\hat{\boldsymbol{x}}$ axis, $u_{x x} \approx \gamma /(\lambda+$ $2 \mu) \equiv \bar{u} \approx 0.1 \%$ where $\lambda \approx 3 \mathrm{eV} \times \AA^{-2}$ and $\mu \approx 9 \mathrm{eV} \times \AA^{-2}$ are the elastic Lamé coefficients of graphene. This strain induces an intervalley gauge field, ${ }^{3,17} A_{x}^{\text {str }}=0, A_{y}^{\text {str }}=\beta \bar{u} / a \approx$ $1.6 \times 10^{-3} \AA^{-1}$, where $a$ is the carbon-carbon distance $(a=$ $a_{0} \approx 1.42 \AA$ at equilibrium $), \beta=-\partial \log (t) / \partial \log (a) \approx 2-$ $3, t$ being the hopping between $\pi$ orbitals in nearest-neighbor carbon atoms ( $t=t_{0} \approx 3 \mathrm{eV}$ at equilibrium). The curvature of the fold hybridizes $\pi$ and $\sigma$ bands, leading to another contribution to the gauge field, ${ }^{18} A_{x}^{\text {hyb }}=0, A_{y}^{\text {hyb }}=\left(3 \epsilon_{\pi \pi} / 8 \hbar v_{\mathrm{F}}\right) \times$ $(a / R)^{2} \approx 7.2 \times 10^{-3} \AA^{-1}$, where $\hbar v_{\mathrm{F}}=3 \mathrm{ta} / 2 \approx 6.4 \mathrm{eV} \times \AA$ 
(a)

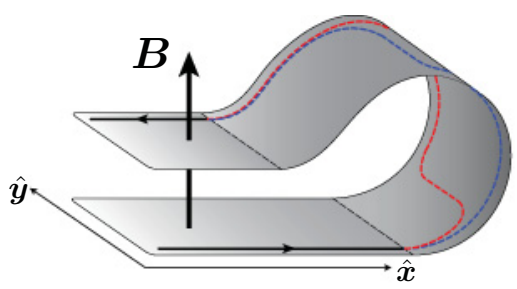

(b)

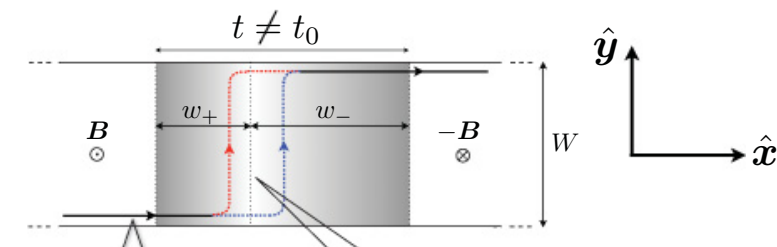

(c)

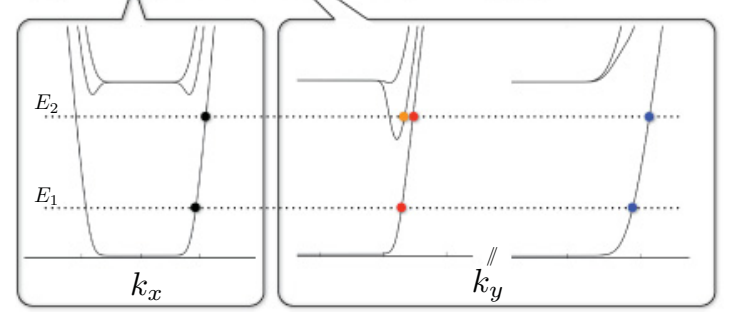

FIG. 1. (Color online) (a) A folded graphene ribbon in a magnetic field. (b) A topologically equivalent geometry obtained by unfolding the ribbon. In this geometry it is clear that the magnetic field has opposite signs in the two layers. In the region of the fold of total length $w=w_{+}+w_{-}$(shaded area) two copropagating snake states (red and blue lines), which are spatially separated due to the deformation-induced gauge field, are present. Note that to have a finite net flux through the region of space delimited by the snake states one needs to have $w_{+} \neq w_{-}$. (c) The low-energy dispersion relations in the asymptotic leads and (d) in the region of the fold are plotted as functions of Bloch momentum $k$ (in units of $\pi / a, a$ being the appropriate lattice constant).

is the Fermi velocity multiplied by $\hbar, \epsilon_{\pi \pi}=V_{p p \pi} / 3+$ $V_{p p \sigma} / 2 \approx 3.0 \mathrm{eV}$, and $V_{p p \pi}, V_{p p \sigma}$ are hoppings between $p$ orbitals in nearest carbon atoms with different orientations. The smallness of the fold radius implies that the contribution from orbital hybridization is dominant.

Let us now consider the folded AGNR in a perpendicular magnetic field $\boldsymbol{B}=B \hat{\boldsymbol{z}}$ [see Fig. 1(a)], strong enough to quantize the single-particle spectrum into well-resolved ${ }^{19}$ Landau levels (LLs) [i.e., we assume that the magnetic length $\ell_{B}=\sqrt{\hbar c /(e B)}$ is much smaller than the width $W$ of the ribbon]. Under these conditions, the current in both layers is carried by edge states, which are localized on opposite sides of the sample for opposite current directions. Upon entering the region of the fold while moving along the transport $(\hat{\boldsymbol{x}})$ direction, the out-of-plane component of the magnetic field first decreases, then becomes zero, and finally changes sign.

Since we are neglecting interlayer hopping, our folded AGNR is topologically equivalent to an unfolded ribbon in the presence of a magnetic field step, as illustrated in Fig. 1(b). Let us now imagine injecting the current from left to right. In this case, the current which is first carried by edge states near the bottom edge of the left layer will have to be carried, after crossing the fold, by edge states on the top edge of the right layer. At low energies (more precisely, at energies below the first LL plateau), only one edge state is involved in carrying this current. In the following we will restrict ourselves to this low-energy "one-channel" regime. Figure 1(c) shows a zoom of the dispersion relation $E=E\left(k_{x}\right)$ of an ideal AGNR in the presence of a quantizing magnetic field. Only the first few low-energy subbands forming the zeroth and the first LLs have been plotted. Note that at sufficiently large values of $\left|k_{x}\right|$ these subbands become dispersive giving rise to the aforementioned quantum-Hall edge states.

Within (or in the proximity of) the region of the fold, a pair of transverse edge modes which live parallel to the axis of the fold (the $\hat{y}$ axis) are induced by the change of sign of the magnetic field. These states are depicted by vertical blue and red lines in Fig. 1(b). Their existence can be understood within a simple semiclassical picture. Similarly to what happens at the sample edges, skipping orbits form in each separate layer close to the $\boldsymbol{B} \mid-\boldsymbol{B}$ interface. These pair of skipping orbits living on the opposite sides of the interface merge together in a pair of so-called "snake states". ${ }^{20}$ In an ideal, flat, $\boldsymbol{B} \mid-\boldsymbol{B}$ interface these two snake states are spatially superimposed and centered on the interface (which we define it to be the line where the $\boldsymbol{B}$ field vanishes).

The effect of the gauge field in the region of the fold is of paramount importance. It shifts the value of the momentum, and, as a result, it changes the position of the LL guiding center. The effective magnetic field has opposite sign for the two valleys, and is maximal in the armchair configuration, while it is zero in the zigzag case. In Sec. III we analyze in detail the edge-state configuration in the proximity of the fold and the eigenfunctions corresponding to the states depicted in Fig. 1(d).

\section{SNAKE STATES}

To understand better the nature of the snake states in the proximity of a $\boldsymbol{B} \mid-\boldsymbol{B}$ interface in the presence of deformations, we introduce an "auxiliary system" consisting of a $\boldsymbol{B} \mid-\boldsymbol{B}$ interface infinitely extended along the $\hat{\boldsymbol{y}}$ direction and of finite length $w_{\text {tot }}$ along the $\hat{\boldsymbol{x}}$ direction (see Fig. 2). We assume that a finite deformation-induced gauge field is present in a strip of total length $w=w_{+}+w_{-} \ll w_{\text {tot }}$. By looking at the spectrum $E\left(k_{y}\right)$ (Bloch momentum $k_{y}$ is a good quantum number because the auxiliary-system interface is infinitely extended along the $\hat{\boldsymbol{y}}$ direction) and at the eigenstates of this auxiliary system we can deduce some quantitative information about the snake states along the $\hat{\boldsymbol{y}}$ direction in our system, which has instead a finite transverse width.

Performing analytical calculations based on the continuum model and numerical ones based on a tight-binding Hamiltonian, we can determine the band structure of such auxiliary system. Figure $1(d)$ shows a low-energy zoom of the dispersion relation $E\left(k_{y}\right)$ as a function of the Bloch momentum $k_{y}$ parallel to the $\boldsymbol{B} \mid-\boldsymbol{B}$ interface. We have selected only the Brillouin-zone portions related to the snake states we are interested in. We immediately observe that the existence of two adjacent regions with opposite magnetic fields modifies the usual LL structure of an ideal ribbon [Fig. 1(c)] by introducing new dispersive branches which correspond to snake modes propagating along the interface. The presence of the gauge field has a crucial role in breaking the symmetry between the two valleys, as it generates a downward bending in the first LL 


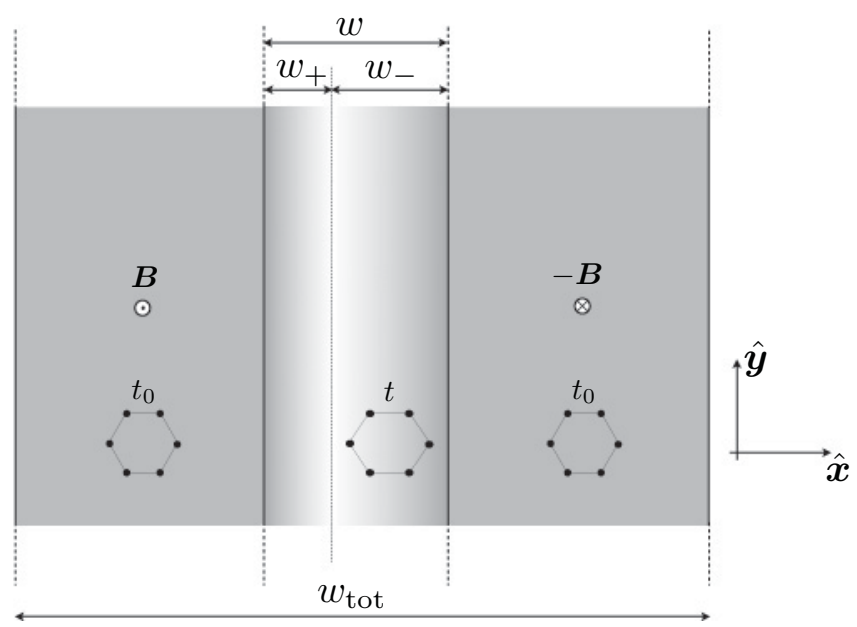

FIG. 2. (Color online) Sketch of the "auxiliary system." It consists of a $\boldsymbol{B} \mid-\boldsymbol{B}$ interface infinitely extended along the $\hat{\boldsymbol{y}}$ direction and of finite length $w_{\text {tot }}$ along the $\hat{\boldsymbol{x}}$ direction. We assume that a finite deformation-induced gauge field is present in a strip of total length $w=w_{+}+w_{-}$, with $w_{+} \neq w_{-}$. This is realized by changing the hopping parameter $t$ along the $\hat{\boldsymbol{x}}$ direction with respect to its equilibrium value $t_{0}$. In the region of width $w_{+}\left(w_{-}\right)$the scalar product between the magnetic field and the oriented normal is positive (negative).

subband of the $K$ valley, say, without modifying the same LL in the $K^{\prime}$ valley.

In Figs. 3 and 4 we present the numerical results for the auxiliary system eigenfunctions [for clarity, in Fig. 3(a) we show again the dispersion curve presented in Fig. 1(d)]. Figures 3(b) through (d) illustrate two-dimensional color plots of the envelope-function profile $\psi_{k_{y}}(x)$ as a function of the Bloch momentum $k_{y}$ and of the coordinate $x$ along the transport direction, for the three types of snake states we are considering. More precisely, Fig. 3(b) shows the wave function belonging to the $K^{\prime}$ valley. For $0.55 \lesssim k_{y} a / \pi \lesssim 0.75$ we have a standard zero-LL edge wave function whose guiding center moves from the right side of the auxiliary system toward the center. For larger values of $k_{y}$ the wave function becomes a snake state localized close to the interface. In Fig. 3(c) we consider instead the $K$ valley, and we plot the wave-function profile corresponding to the lowest-energy subband. Again, for small values of $k_{y}$ we find ordinary edge states with a guiding center that moves from the left side of the auxiliary system toward the center. This is then converted into a snake state which approaches the interface asymptotically. It is crucial to observe that the "centers" of the two snake states corresponding to the two valleys are not superimposed but are instead displaced by a finite amount. Finally, in Fig. 3(d) we have plotted the wave function $\psi_{k_{y}}(x)$ of the states belonging to the first LL. Since this is an excited subband, the corresponding wave functions have a node, and thus the probability amplitude $\left|\psi_{k_{y}}(x)\right|^{2}$ presents a double-peaked structure in space, centered around the $\boldsymbol{B} \mid-\boldsymbol{B}$ interface.

More explicit plots of the auxiliary system eigenfunctions are shown in Fig. 4, which contains typical one-dimensional cuts of $\psi_{k_{y}}(x)$ for a fixed value of $k_{y}$. More precisely, Fig. 3(a) reports the squared modulus $\left|\psi_{k_{y}}(x)\right|^{2}$ of the $A$-sublattice wave function corresponding to a snake state in the lowest-energy subband. The spatial region where deformations are present (here taken to be 6-nm wide) is inside the area delimited by the two vertical lines. The position where the $\boldsymbol{B}$ field vanishes is not shown in this plot for simplicity but lies within this region. The value of $k_{y}$ chosen to make this plot corresponds to an energy intermediate between the zero and the first LL. As shown in Fig. 3, one of these two snake states belongs to the $K$ valley, while the other one belongs to the $K^{\prime}$ valley. From Fig. 4(a) it is very clear that the centers of the two snake states are slightly shifted with respect to each other by roughly $10 \mathrm{~nm}$.

From theoretical estimates the spatial separation $\delta_{x}$ between the two snake states is given by

$$
\delta_{x} \approx \min \left(A_{y}^{\mathrm{hyb}} \ell_{B}^{2}, \pi R\right),
$$

with $A_{y}^{\text {hyb }} \ell_{B}^{2} \approx 100 \mathrm{~nm} / B[\mathrm{~T}]$ for the parameters described earlier. For realistic values of the magnetic field the separation between edge states is thus of the order of the length $\pi R$ of the folded region, and the carriers near the fold are valley

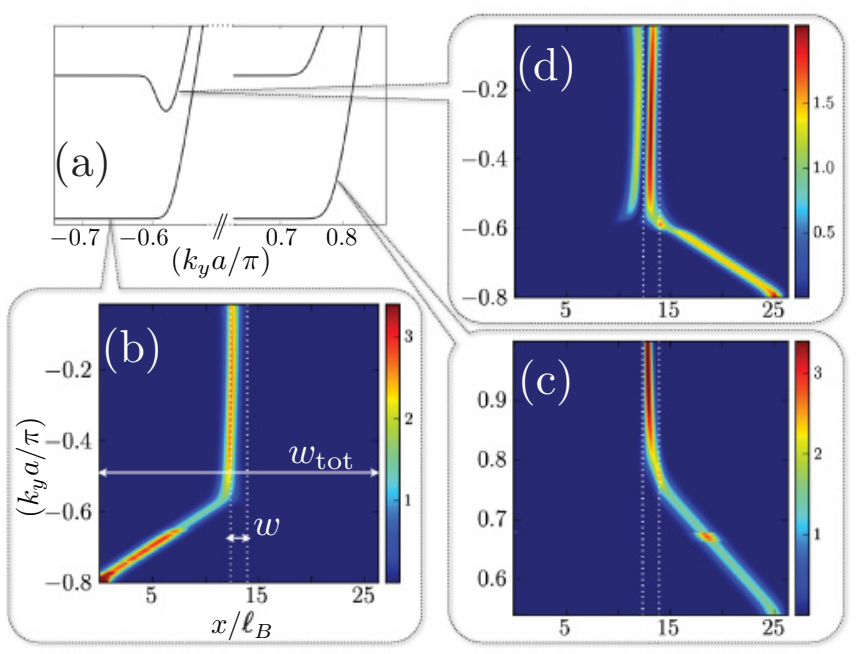

FIG. 3. (Color online) (a) A zoom of the dispersion relation of the auxiliary system (see main text) in the vicinity of the Dirac points. (b) Two-dimensional color plot of the $K$-valley squared wave functions $\left|\psi_{k_{y}}(x)\right|^{2}$ of the auxiliary system (only the value of the wave function on one type of sublattice, say the $A$ type, is shown). In the horizontal axis the spatial position $x$ is shown in units of the magnetic length $\ell_{B}$. In the vertical axis we plot the Bloch momentum $k_{y}$ parallel to the interface. (c) Same as in (b), but for the $K^{\prime}$ valley. (d) Same as in the previous panels, but for the first-excited subband, which includes the first LL and the downward dip induced by the gauge field (only the $K$ valley is considered, the $K^{\prime}$ valley has no dip). 


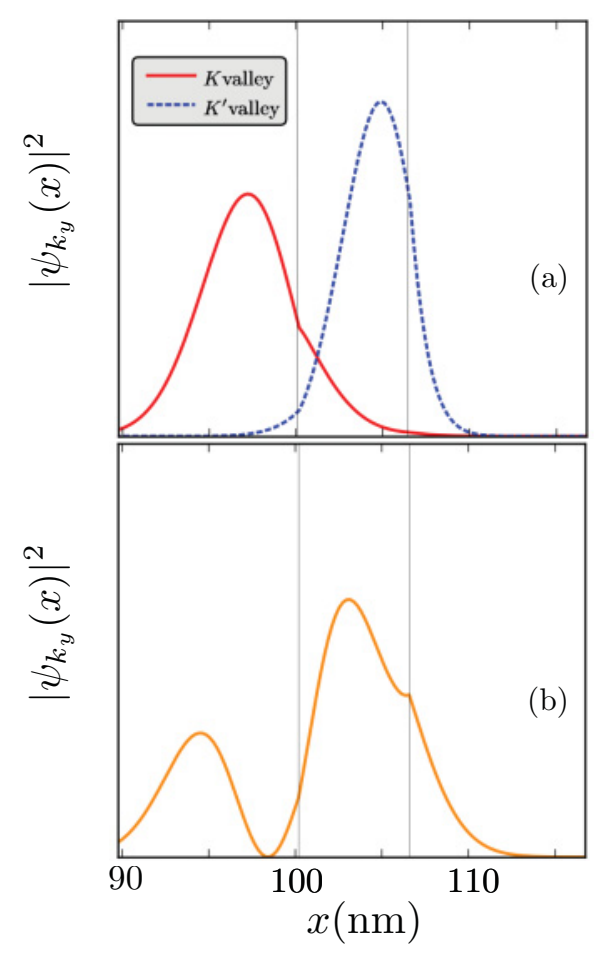

FIG. 4. (Color online) Wave functions in the $\boldsymbol{B} \mid-\boldsymbol{B}$ interface in the presence of deformations. (a) Probability density $\left|\psi_{k_{y}}(x)\right|^{2}$ for the snake states residing along the $\boldsymbol{B} \mid-\boldsymbol{B}$ interface. The horizontal axis is a zoom around the center of the 207-nm-wide auxiliary system, where the interface is located. Data represented by the solid (dashed) line refer to the $K\left(K^{\prime}\right)$ valley. The two vertical thin lines define the deformed region within which the $\boldsymbol{B} \rightarrow-\boldsymbol{B}$ transition takes place. We have chosen an intermediate energy value in the one-channel regime, while the magnetic field is $B=10 \mathrm{~T}$. (b) Probability density $\left|\psi_{k y}(x)\right|^{2}$ for the snake state belonging to the first-excited subband [see Fig. 3(d)]. Notice that this single eigenstate is characterized by a double-peak probability distribution, which could generate interference effects similar to the ones occurring in an Aharonov-Bohm ring interferometer.

polarized. This separation prevents short-range scattering from mixing the valleys, ${ }^{21}$ unlike in other proposals to achieve valley polarization in graphene. The number of flux quanta in the region between the two channels, using the previous parameters, is

$$
N_{\text {flux }} \approx \zeta \frac{(\pi R) W B}{\Phi_{0}} \approx \zeta \frac{W[\mathrm{~nm}] B[\mathrm{~T}]}{1800},
$$

where $\zeta=\left|w_{+}-w_{-}\right| / w$ is a dimensionless parameter defining the asymmetry of the fold [see Fig. 1(b)], $\Phi_{0} \approx 4.1 \times$ $10^{3} \mathrm{~T} \times \mathrm{nm}^{2}$ is the magnetic flux quantum, and we are neglecting a factor proportional to the angle between the field and the local orientation of the layer.

The flux in Eq. (2) allows for the operation of the device in Fig. 1(a) as an interferometer. Incoming electrons propagating along the bottom edge state in the left layer, say, can either be transmitted to copropagating edge states on the right layer or be reflected back. In both cases, the outgoing edge state is localized on the top edge of the sample. Electrons can move from one side to the other thanks to the existence of the snake states discussed above. Due to the gauge fieldinduced separation $\delta_{x}$, the two snake state trajectories enclose a finite magnetic flux, Eq. (2). If the deformed $\boldsymbol{B} \mid-\boldsymbol{B}$ interface provides a finite coupling between the two snake states, the enclosed residual flux manifests itself as a phase difference between the two quantum trajectories involving the $K$ and $K^{\prime}$ interface snake states.

Another appealing possibility of interference is offered by the snake state depicted in Fig. 4(b), which belongs to the first subband of the $K$ valley. As we have already pointed out, this state presents a node close to the interface, and thus the corresponding probability density $\left|\psi_{k_{y}}(x)\right|^{2}$ possesses a double-peak structure. In this case thus there is no need of an intervalley coupling mechanism to observe interference. An incoming electron can split at the interface into a twopath configuration, which can generate Aharonov-Bohm-type interference analogous to the one seen in ordinary quantum rings.

\section{NUMERICAL RESULTS}

We now turn to present our main numerical results for the differential conductance $G$ of a folded AGNR in a strong magnetic field and in the presence of deformations in the region of the fold. Our calculations, which rely on the Landauer formalism, are based on the tight-binding description and on the recursive Green's function technique. ${ }^{22}$

In Fig. 5 we present data for $G$ as a function of the applied magnetic field $B$. The simulated ribbon has a width $W \approx 100 \mathrm{~nm}$ : for this size the quantum Hall regime is reached at $B \gtrsim 10$ T. The length $w$ of the folded region, in which the gauge field is active, is taken to be $w \approx 30 \mathrm{~nm}$. We describe the total gauge field $\boldsymbol{A}=\boldsymbol{A}^{\mathrm{str}}+\boldsymbol{A}^{\text {hyb }}$ as a modulation of the hopping parameter, although it is mostly due to the hybridization of the $\pi$ and $\sigma$ bands (see Supplementary Information). This description preserves the symmetries and main features of the gauge field, and should not change the results significantly. The modulation of the hoppings parallel to the $\hat{\boldsymbol{x}}$ axis, $\delta t / t_{0} \equiv\left(t-t_{0}\right) / t_{0}$, varies between $3 \%$ [Fig. $\left.5(\mathrm{a})\right]$ and $10 \%$ [Fig. 5(b)]. These values are higher than those expected in a realistic fold. They allow us to illustrate the combined effect of gauge potentials and magnetic fields in ribbons of widths amenable to numerical analysis. A lower gauge field will give similar results in wider ribbons, see Eqs. (1) and (2).

The most important results of this work are the data represented by filled circles in Fig. 5. They clearly show an oscillatory behavior of the conductance $G$ as the magnetic field is varied. We emphasize that these oscillations are only due to the presence of a gauge field in the region of the fold. ${ }^{23}$ To make this more evident, in Fig. 5 we have also reported the conductance of the same $\boldsymbol{B} \mid-\boldsymbol{B}$ interface in the absence of deformations (filled triangles). We can clearly see how the conductance oscillations disappear when the gauge field is switched off. In this case, one can see that $G$ is always equal to the maximum conductance $2 e^{2} / h$ (in the one-channel regime). This is part of a more general result: Due to symmetry properties of the zeroth LL, a folded graphene nanoribbon in the absence of a gauge field shows always perfect transmission when the current is carried by the zeroth edge state. ${ }^{12}$ 

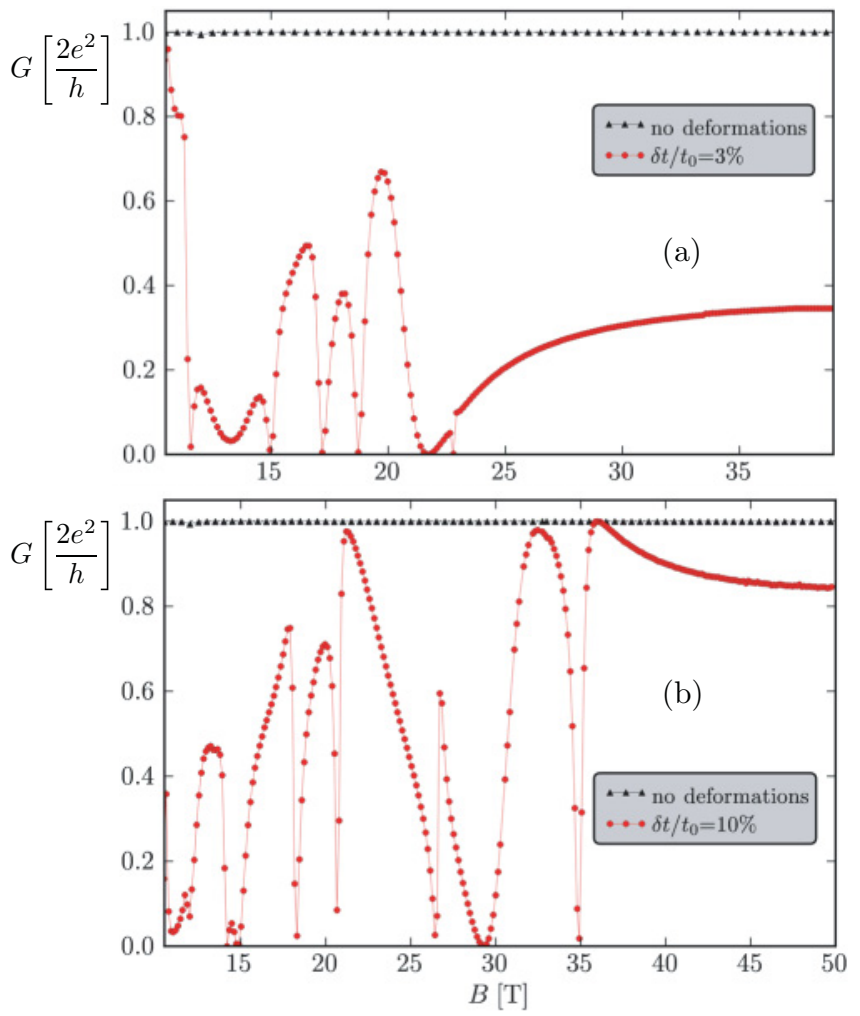

FIG. 5. (Color online) (a) Numerical results for the two-terminal conductance $G$ (in units of $2 e^{2} / h$ ) of the device in Fig. 1(a) as a function of the applied magnetic field (in T). In these simulations $W \approx 100 \mathrm{~nm}$ and $w \approx 30 \mathrm{~nm}$. The chemical potential is located at $\approx 100 \mathrm{meV}$ above the Dirac level. The data labeled by filled (red) circles refer to the case of a folded AGNR with a modified hopping integral $t / t_{0}=0.97$ along the $\hat{\boldsymbol{x}}$ axis in the region of the fold. The data labeled by filled (black) triangles refer to the case of a folded AGNR in the absence of deformations $\left(t / t_{0}=1.0\right.$ everywhere). (b) Same as in (a) but the data labeled by filled (red) circles have now been obtained for a larger value of $\delta t / t_{0}$, i.e. $t / t_{0}=0.90$.

Some comments related to the data in the presence of gauge fields are now in order.

(1) The behavior of the conductance as a function of the $B$ field is roughly characterized by two quite distinct regimes. We can identify a region of type "I," approximately comprised between 10 and $15 \mathrm{~T}$ in Fig. 5 (a) and between 10 and $26 \mathrm{~T}$ in Fig. 5(b), and a region of type "II" for $B \gtrsim 15 \mathrm{~T}$ in Fig. 5(a) $[B \gtrsim 26 \mathrm{~T}$ in Fig. 5(b)]. (For $B \lesssim 10 \mathrm{~T}$ one exits the one-channel regime.) In both regions the conductance $G(B)$ shows clear oscillations. In region II and for sufficiently large values of the magnetic field the conductance changes slowly and eventually saturates. One can show that type-II oscillations are generated by interference between the two snake states in Fig. 4(a), whereas type-I oscillations are caused by the existence of the double-peaked snake state shown in Fig. 4(b). Conductance oscillations of type II typically have a larger amplitude than those of type I.

(2) Comparing the data in Fig. 5(b) with those in Fig. 5(a), we notice that a smaller gauge field produces oscillations with smaller amplitude. Moreover, for larger gauge fields the type-II behavior is reached at larger values of the magnetic field.

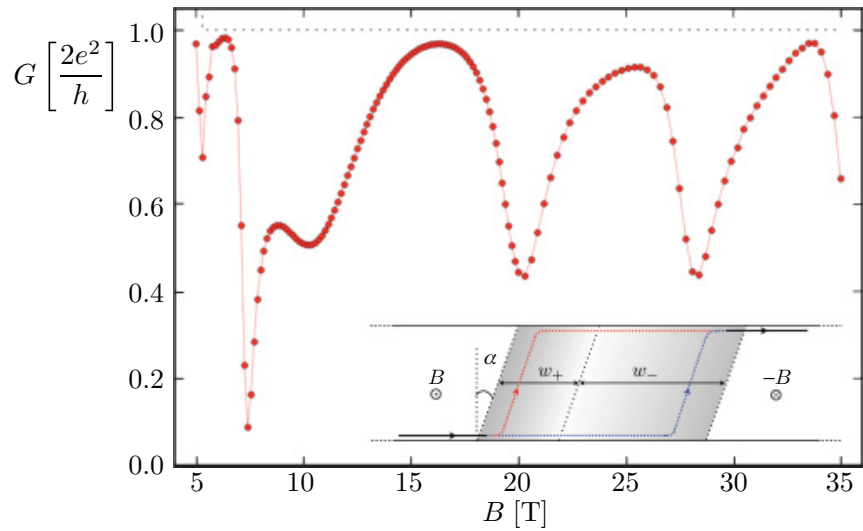

FIG. 6. (Color online) The conductance of an armchair folded graphene nanoribbon with width $W \approx 100 \mathrm{~nm}$. These results are for an oblique fold (see inset) with $\alpha=12^{\circ}$. The total length of the folded region is $w=\left(w_{+}+w_{-}\right) \approx 6 \mathrm{~nm}$. The chemical potential has been set at $\approx 75 \mathrm{meV}$ above the Dirac level. The modulation of the hopping in the folded region is $\delta t / t_{0}=3 \%$.

Finally, both regions are characterized by sharp antiresonances, which occur since the fold (where snake-states are present) acts as a "t-stub" resonator. ${ }^{24}$

\section{A. Numerical results for oblique folds}

Above we have considered the ideal situation of a fold orthogonal to the ribbon axis. However, the two graphene layers of a folded graphene sheet can be found in a more general relative angular position, specified by an angle $\alpha$ that we have defined in the inset to Fig. 6. Orthogonal folds as those studied above correspond to $\alpha=0$. In Fig. 6 we show a typical result for the conductance as a function of the applied magnetic field for an "oblique fold." We clearly see how well-defined conductance oscillations exist in this case as well.

\section{B. Numerical results in the presence of structural disorder at the edges}

We finally study the role of structural disorder at the edges of an armchair folded graphene nanoribbon. We implement disorder by removing a certain number of atoms in the two outmost rows of the ribbon. In Fig. 7 we show numerical results for a removal probability $p=25 \%$ and two transverse widths: $W \approx 50 \mathrm{~nm}$ [Fig. 7(a)] and $W \approx 100 \mathrm{~nm}$ [Fig. 7(b)]. These results have been obtained for two specific realizations of disorder.

In the absence of a deformation-induced gauge field in the folded region, ${ }^{12}$ we clearly see how the clean-system result $G=2 e^{2} / h$ is spoiled by disorder (data labeled by black filled triangles in Fig. 7). On the contrary, conductance oscillations survive the presence of disorder, provided that the applied magnetic field is not too large (data labeled by red filled circles in Fig. 7).

Finally, an analysis of the role of armchair versus zigzag boundaries is summarized in Appendix B. 

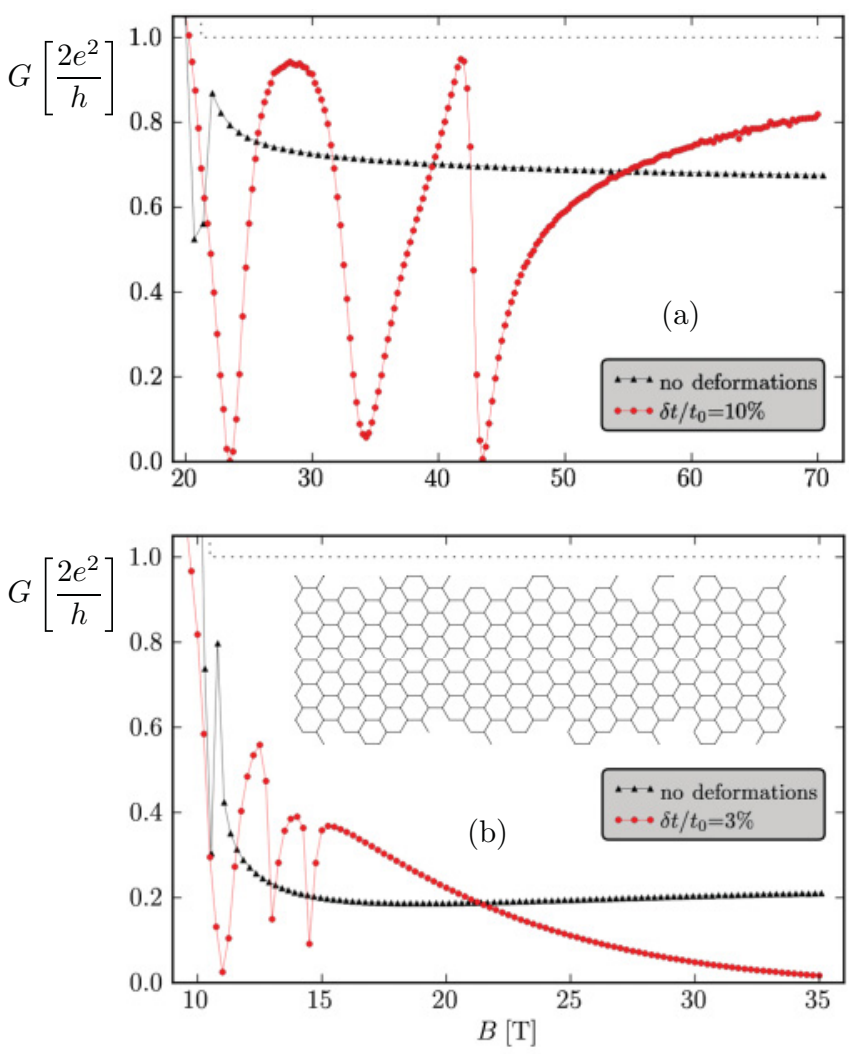

FIG. 7. (Color online) Effect of structural disorder at the edges on the conductance oscillations through a folded graphene nanoribbon. In both panels the removal probability is $p=25 \%$ and data labeled by black filled triangles refer to the situation in which no deformation-induced gauge field is present in the region of the fold. (a) Conductance as a function of the applied magnetic field for a nanoribbon with a width $W \approx 50 \mathrm{~nm}$ and $w \approx 6 \mathrm{~nm}$. The chemical potential has been set at $\approx 150 \mathrm{meV}$ above the Dirac level and the modulation of the hopping parameter is $\delta t / t_{0}=10 \%$. (b) Same as in (a) but for $W \approx 100 \mathrm{~nm}$ and $w \approx 10 \mathrm{~nm}$. The chemical potential has been set at $\approx 100 \mathrm{meV}$ above the Dirac level and the modulation of the hopping parameter is $\delta t / t_{0}=3 \%$. The inset to panel (b) illustrates the type of structural disorder we have considered.

\section{CONCLUSIONS}

In this work we have considered two-terminal conductance measurements on a folded graphene layer in the Hall regime, with the assumption that the two layers can be contacted separately. We argue that the combination of magnetic field and fold-induced gauge fields result in a peculiar edge-state configuration in the region of the fold. Such configuration enables interferometry experiments thanks to the finite magnetic flux enclosed between the two possible paths that an electron can follow while being transmitted from one layer to the other. By means of numerical techniques based on the recursive Green's function method, we corroborate our predictions, showing that the calculated conductance oscillates as a function of the externally applied magnetic field.

The analysis can be extended in a straightforward way to multiterminal setups, where more complex correlations can be monitored. The combination of flexibility and stiffness of graphene allows for many other combinations of gauge fields and electronic currents.

\section{ACKNOWLEDGMENTS}

Work in Pisa and in Madrid was supported by the 2009/2010 CNR-CSIC scientific cooperation project. F.G. is supported by MICINN (Spain), Grants No. FIS2008-00124 and No. CONSOLIDER CSD2007-00010. M.P., F.G., and V.F. also acknowledge partial financial support by the Project "Knowledge Innovation Program" (PKIP) of the Chinese Academy of Sciences, Grant No. KJCX2.YW.W10. D.R., F.T., and M.P. wish to thank Rosario Fazio for numerous enlightening discussions.

\section{APPENDIX A: ELASTIC STRAINS AND GAUGE FIELDS}

The elastic energy of a graphene layer near a planar configuration can be written as ${ }^{25}$

$$
\begin{aligned}
E_{\mathrm{el}}= & \int d^{2} \boldsymbol{r}\left\{\frac{\lambda}{2}\left[u_{x x}(\boldsymbol{r})+u_{y y}(\boldsymbol{r})\right]^{2}\right. \\
& \left.+\mu\left[u_{x x}^{2}(\boldsymbol{r})+u_{y y}^{2}(\boldsymbol{r})+2 u_{x y}^{2}(\boldsymbol{r})\right]\right\} \\
& +\frac{\kappa}{2} \int d^{2} \boldsymbol{r}\left[\partial_{x x} u_{z}(\boldsymbol{r})+\partial_{y y} u_{z}(\boldsymbol{r})\right]^{2},
\end{aligned}
$$

where $u_{i j}$ is the deformation tensor defined by ${ }^{25}$

$$
\begin{gathered}
u_{x x}=\partial_{x} u_{x}+\frac{\left(\partial_{x} u_{z}\right)^{2}}{2}, \\
u_{y y}=\partial_{y} u_{y}+\frac{\left(\partial_{y} u_{z}\right)^{2}}{2}, \\
u_{x y}=\frac{\partial_{x} u_{y}+\partial_{y} u_{x}}{2}+\frac{\partial_{x} u_{z} \partial_{y} u_{z}}{2},
\end{gathered}
$$

$u_{i}$ being atomic displacement vectors. The parameters $\lambda \approx$ $3 \mathrm{eV} \times \AA^{2}$ and $\mu \approx 9 \mathrm{eV} \times \AA^{2}$ are the Lamé coefficients of graphene, while $\kappa \approx 1 \mathrm{eV}$ is the bending rigidity. ${ }^{26}$ The van der Waals attraction energy between the graphene layers is

$$
E_{\mathrm{vdW}}=\gamma \int_{\Omega} d^{2} \boldsymbol{r},
$$

where $\Omega$ is the contact region, which does not include the fold, and $\gamma \approx 0.022 \mathrm{eV} \times \AA^{2}$ is the van der Waals interaction. ${ }^{27}$

To a first approximation, the radius of the fold $R$ is determined by the balance between the bending rigidity and the van der Waals interaction. ${ }^{15}$ Approximating the fold by half a cylinder of radius $R$ we find

$$
R \approx \sqrt{\frac{\kappa}{\gamma}} \approx 7 \AA .
$$

The presence of a substrate and the finite separation between the layers, $d \approx 3.3 \AA$, tend to increase this value and make the shape asymmetric. The distribution of curvatures and strains (see below) will become inhomogeneous and asymmetric as well. 


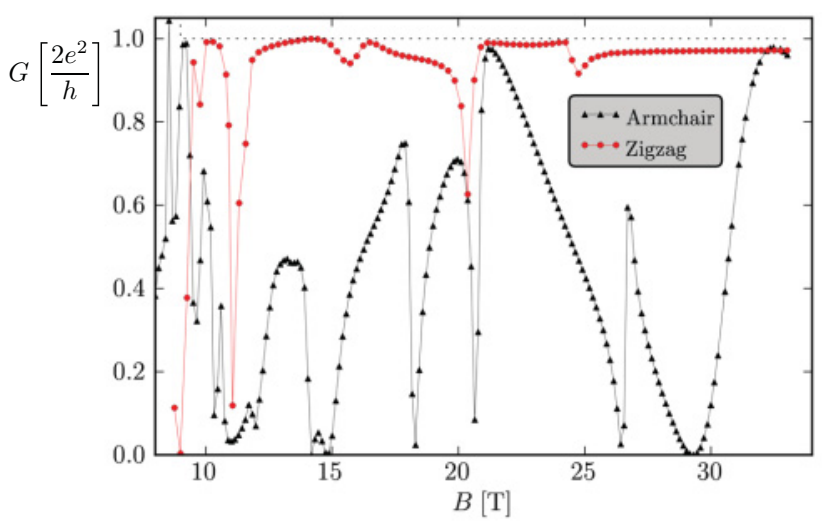

FIG. 8. (Color online) Conductance of a folded graphene nanoribbon with zigzag edges along the transport $(\hat{\boldsymbol{x}})$ direction (red filled circles). The parameters used to produce this plot are $W \approx 100 \mathrm{~nm}$, $w \approx 20 \mathrm{~nm}$, and $\delta t / t_{0}=10 \%$. The chemical potential is located at $\approx 100 \mathrm{meV}$ above the Dirac level. For the sake of comparison we have also reported data for an identical ribbon with armchair edges. It is evident that in the case of zigzag edges no regular and distinct oscillations are present, the conductance being almost always equal to unity apart from some antiresonances.

Now, the bending energy is reduced by an expansion of the half cylinder, which leads to in-plane strains. Assuming a constant uniaxial strain $u$ we find

$$
E_{\mathrm{el}}(R)=\frac{\pi R \kappa}{R^{2}(1+u)}+\frac{\pi R(\lambda+2 \mu) u^{2}}{2} .
$$

Minimizing this expression with respect to $u$ and using Eq. (A4), we finally find

$$
u \approx \frac{\kappa}{(\lambda+2 \mu) R^{2}} \approx \frac{\gamma}{(\lambda+2 \mu)} \equiv \bar{u} \approx 1.0 \times 10^{-3} .
$$

A similar result has been found for carbon nanotubes. ${ }^{28}$ In the folded region, this strain leads to a constant gauge field ${ }^{3,17,29}$

$$
\left\{\begin{array}{l}
A_{x}^{\mathrm{str}}=0 \\
A_{y}^{\mathrm{str}} \equiv \frac{\beta \bar{u}}{a} \approx 1.4 \times 10^{-3} \AA^{-1},
\end{array}\right.
$$

where $a$ is the distance between nearest neighbors in the graphene lattice $\left(a=a_{0} \approx 1.42 \AA\right.$ at equilibrium), $\beta=$ $-\partial \log (t) / \partial \log (a) \approx 2-3$, and $t$ is the hopping between $\pi$ orbitals in nearest neighbor atoms $\left(t=t_{0} \approx 3 \mathrm{eV}\right.$ at equilibrium). This situation has been considered in Ref. 30. In the absence of a magnetic field, the transmission can be calculated analytically.

The bending of the layer induces the hybridization of $\pi$ and $\sigma$ orbitals, and the emergence of a new contribution to the total gauge field, not related to strains. ${ }^{18}$ Its value is

$$
\left\{\begin{array}{l}
A_{x}^{\text {hyb }}=0 \\
A_{y}^{\text {hyb }} \equiv \frac{3 \epsilon_{\pi \pi}}{8 \hbar v_{\mathrm{F}}} \frac{a^{2}}{R^{2}} \approx 7.2 \times 10^{-3} \AA^{-1},
\end{array}\right.
$$

where $v_{\mathrm{F}}$ is the Fermi velocity, $\hbar v_{\mathrm{F}}=3 \mathrm{ta} / 2 \approx 6.4 \mathrm{eV} \times \AA$, and $\epsilon_{\pi \pi}=\left(V_{p p \sigma} / 2+V_{p p \pi} / 3\right) \approx 3.0 \mathrm{eV}, V_{p р \pi}$ and $V_{p p \sigma}$ being the two possible hopping integrals between $p$ orbitals in neighboring carbon atoms. Due to the smallness of the radius of the fold, the contribution from the hybridization in Eq. (A8) is roughly one order of magnitude larger than the one due to strains, given in Eq. (A7). It is equivalent to a relative change of the value of the hopping $t$ of $1 \%$ (taking $\beta=2$ ).

\section{APPENDIX B: INFLUENCE OF LATTICE ORIENTATION}

As already shown in Fig. 6 of the main text, the conductance oscillations discussed above are absent when the gauge field is switched off. Our results then are similar to those reported in Ref. 12 (see black filled triangles in Fig. 6 of the main text).

A similar disappearance of the oscillations occurs also if the orientation of the lattice with respect to the fold axis is rotated by $90^{\circ}$ (zigzag edges along the transport direction). The gauge field in this case is $\boldsymbol{A}=A(x) \hat{\boldsymbol{x}} \propto \Theta(w / 2-|x|) \hat{\boldsymbol{x}}$. Such a pseudovector potential has zero curl (i.e., it can be gauged away). We thus expect that the oscillations disappear in this case: This is indeed confirmed by our numerical calculations, as illustrated in Fig. 8. It is interesting to note that the gauge transformation is applicable only to the effect of the gauge field on the electronic wave functions. The lattice deformations which originate the gauge field, obviously, cannot be canceled.

\footnotetext{
*diego.rainis@unibas.ch; Present address: Department of Physics, University of Basel, Klingelbergstrasse 82, 4056 Basel, Switzerland.

${ }^{1} \mathrm{~F}$. Wilczek, The Lightness of Being (Basic-Perseus, New York, 2008).

${ }^{2}$ A. H. Castro Neto, F. Guinea, N. M. R. Peres, K. S. Novoselov, and A. K. Geim, Rev. Mod. Phys. 81, 109 (2009).

${ }^{3}$ M. A. H. Vozmediano, M. I. Katsnelson, and F. Guinea, Phys. Rep. 496, 109 (2010).

${ }^{4}$ F. Guinea, M. I. Katsnelson, and A. K. Geim, Nature Phys. 6, 30 (2010).

${ }^{5}$ N. Levy, S. A. Burke, K. L. Meaker, M. Panlasigui, A. Zettl, F. Guinea, A. H. Castro Neto, and M. F. Crommie, Science 329, 544 (2010).
}

${ }^{6}$ C. Lee, X. Wei, J. W. Kysar, and J. Hone, Science 321, 385 (2008); T. J. Booth, P. Blake, R. R. Nair, D. Jiang, E. W. Hill, U. Bangert, A. Bleloch, M. Gass, K. S. Novoselov, M. I. Katsnelson, and A. K. Geim, Nano Lett. 8, 2442 (2008); J. S. Bunch, S. S. Verbridge, J. S. Alden, A. M. van der Zande, J. M. Parpia, H. G. Craighead, and P. L. McEuen, ibid. 8, 2458 (2008).

${ }^{7}$ M. Huang, H. Yan, C. Chen, D. Song, T. F. Heinz, and J. Hone, Proc. Natl. Acad. Sci. USA 106, 7304 (2009); T. M. G. Mohiuddin, A. Lombardo, R. R. Nair, A. Bonetti, G. Savini, R. Jalil, N. Bonini, D. M. Basko, C. Galiotis, N. Marzari, K. S. Novoselov, A. K. Geim, and A. C. Ferrari, Phys. Rev. B 79 , 205433 (2009); W. Bao, F. Miao, Z. Chen, H. Zhang, W. Jang, C. Dames, and C. N. Lau, Nature Nanotech. 4, 562 (2009). 
${ }^{8}$ Z. Ni, Y. Wang, T. Yu, Y. You, and Z. Shen, Phys. Rev. B 77, 235403(2008); P. Poncharal, A. Ayari, T. Michel, and J.-L. Sauvajol, ibid. 78, 113407 (2008); Z. Liu, K. Suenaga, P. J. F. Harris, and S. Iijima, Phys. Rev. Lett. 102, 015501 (2009); J. Y. Huang, F. Ding, B. I. Yakobson, P. Lu, L. Qi, and J. Li, Proc. Natl. Acad. Sci. USA 106, 10103 (2009).

${ }^{9}$ H. Schmidt, T. Lüdtke, P. Barthold, E. McCann, V. I. Fal'ko, and R. J. Haug, Appl. Phys. Lett. 93, 172108 (2008); H. Schmidt, T. Lüdtke, P. Barthold, and R. J. Haug, Phys. Rev. B 81, 121403(R) (2010).

${ }^{10}$ M. Grayson, L. Steinke, D. Schuh, M. Bichler, L. Hoeppel, J. Smet, K. v. Klitzing, D. K. Maude, and G. Abstreiter, Phys. Rev. B 76, 201304(R) (2007).

${ }^{11}$ Y. Ji, Y. Chung, D. Sprinzak, M. Heiblum, D. Mahalu, and H. Shtrikman, Nature (London) 422, 415 (2003); I. Neder, M. Heiblum, Y. Levinson, D. Mahalu, and V. Umansky, Phys. Rev. Lett. 96, 016804 (2006); L. V. Litvin, H.-P. Tranitz, W. Wegscheider, and C. Strunk, Phys. Rev. B 75, 033315 (2007); I. Neder, N. Ofek, Y. Chung, M. Heiblum, D. Mahalu, and V. Umansky, Nature (London) 448, 333 (2007).

${ }^{12}$ E. Prada, P. San-Jose, and L. Brey, Phys. Rev. Lett. 105, 106802 (2010).

${ }^{13}$ It is very unlikely to have perfect commensurability between honeycomb lattices on the two layers of a folded graphene sheet. Even though they can be atomically close, a mismatch with respect to $\mathrm{AB}$ or $\mathrm{AA}$ stacking strongly suppresses interlayer hopping: see, for example, A. Luican et al., e-print arXiv:1010.4032 and references therein.

${ }^{14}$ Interlayer electron-electron interactions, which have been neglected in the present work, are expected to have merely quantitative effects on the conductance oscillations we discuss below. They might indeed reduce their visibility.

${ }^{15}$ S. Cranford, D. Sen, and M. J. Buehler, Appl. Phys. Lett. 95, 123121 (2009).
${ }^{16}$ J. Zhang, J. Xiao, X. Meng, C. Monroe, Y. Huang, and J.-M. Zuo, Phys. Rev. Lett. 104, 166805 (2010).

${ }^{17}$ H. Suzuura and T. Ando, Phys. Rev. B 65, 235412 (2002).

${ }^{18}$ E.-A. Kim and A. H. Castro Neto, Europhys. Lett. 84, 57007 (2008).

${ }^{19}$ Due to the massless character of its carriers, LLs in graphene are well resolved even at room temperature: K. S. Novoselov, Z. Jiang, Y. Zhang, S. V. Morozov, H. L. Stormer, U. Zeitler, J. C. Maan, G. S. Boebinger, P. Kim, and A. K. Geim, Science 315, 1379 (2007).

${ }^{20}$ L. Oroszlány, P. Rakyta, A. Kormányos, C. J. Lambert, and J. Cserti, Phys. Rev. B 77, 081403(R) (2008); T. K. Ghosh, A. De Martino, W. Häusler, L. Dell' Anna, and R. Egger, ibid. 77, 081404(R) (2008).

${ }^{21}$ We stress, however, that electronic wave functions centered on the $A$ sublattice of the $K$ valley, say, have a finite overlap with wave functions centered on the $B$ sublattice of the $K^{\prime}$ valley.

${ }^{22}$ See, for example, D. Rainis, F. Taddei, F. Dolcini, M. Polini, and R. Fazio, Phys. Rev. B 79, 115131 (2009) and references therein.

${ }^{23}$ We have checked that the conductance oscillations in Fig. 5 are not due to the rapidity with which the gauge field is switched on in the region of the fold.

${ }^{24}$ Z.-A. Shao, W. Porod, and C. S. Lent, Phys. Rev. B 49, 7453 (1994).

${ }^{25}$ L. D. Landau, L. P. Pitaevskii, E. M. Lifshitz, and A. M. Kosevich, The Theory of Elasticity (Butterworth-Heinemann, Oxford, 1986).

${ }^{26}$ K. V. Zakharchenko, M. I. Katsnelson, and A. Fasolino, Phys. Rev. Lett. 102, 046808 (2009).

${ }^{27}$ L. Spanu, S. Sorella, and G. Galli, Phys. Rev. Lett. 103, 196401 (2009).

${ }^{28}$ D. Sánchez-Portal, E. Artacho, J. M. Soler, A. Rubio, and P. Ordejón, Phys. Rev. B 59, 12678 (1999).

${ }^{29}$ J. L. Mañes, Phys. Rev. B 76, 045430 (2007).

${ }^{30}$ M. M. Fogler, F. Guinea, and M. I. Katsnelson, Phys. Rev. Lett. 101, 226804 (2008). 